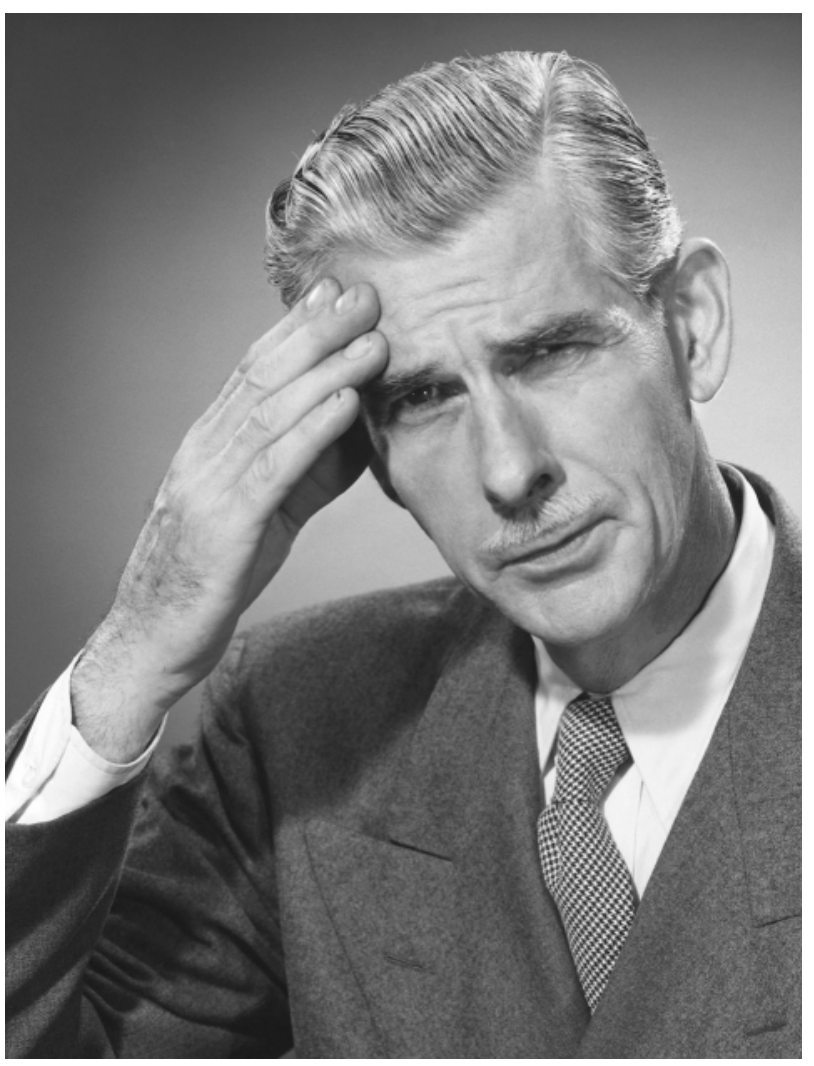

T-CELL DEVELOPMENT

\section{The ageing process}

The appearance of grey hairs is just one sign that a person is getting older. Changes within our immune system also occur as we age. The thymus - the site of T-cell development - shrinks or involutes from adolescence onwards. Until now, the molecular signals involved in the maintenance of the thymus have been unclear. Work by Sano and colleagues in Immunity now shows an essential role for signal transducer and activator of transcription 3 (Stat3) in these processes.

Interactions between thymic stromal cells and lymphoid cells are vital for the formation and maintenance of thymic structure. Stat3, a transcriptional regulator involved in growthfactor signalling, might be important in these processes. As Stat $3^{-1-}$ mice die mid-gestation, the authors used Cre/loxP technology to investigate the role of Stat3 in the maintenance of thymic architecture. They crossed Stat3 floxed mice with transgenics that expressed Cre recombinase under the control of the keratin 5 (K5) promoter, so that Stat3 was exclusively deleted in stratified epithelial cells. This is the first time this technique has been used to successfully delete a gene from thymic epithelial cells (TECs) without affecting the genomes of the thymocytes. At birth thymocyte development was normal in these mice, but from adolescence onwards the numbers of thymocytes in the Stat $3^{-1-}$ mice decreased significantly in comparison with wild-type littermates. This thymic hypoplasia worsened with age, as did the observed changes in cortical TEC architecture.

Stat 3 activates the expression of anti-apoptotic proteins, so the authors next asked whether the agedependent thymic hypoplasia resulted from increased apoptosis. They found more apoptotic thymocytes in

\title{
Decisions, decisions
}

T cell or B cell - what's a lymphoid progenitor to do? The molecular basis of this fundamental cell-fate decision is not known, although there are indications that Notch signalling might be involved. Reporting in Immunity, Koch and co-workers provide further support for the role of Notch1 in the T-versus B-cell-fate decision, and show that lunatic fringe, a Notch regulator, can influence this choice - but in an unexpected and novel way.

Notch proteins are evolutionarily conserved receptors that are known to direct cell-fate decisions in a variety of developmental settings. In the absence of Notch1, B-cell development is promoted at the expense T-cell development, and B cells are found in the thymus. Conversely, in the presence of a constitutively active Notch1, B-cell development is blocked, and T cells develop in the bone marrow. These studies imply that Notch 1 expression normally blocks B-cell commitment. But Notch proteins do not act alone. The fringe family are Golgi-resident proteins that are thought to modulate Notch signalling in a cellautonomous manner by modifying the Notch receptors.

One fringe protein, lunatic fringe, is expressed at high levels in the thymic medulla but not in the thymic cortex, in which immature thymocytes are found. The authors constructed transgenic mice expressing lunatic fringe under the control of the $556^{\text {lck }}$ proximal-promoter, which have high levels of lunatic fringe in the thymic cortex. These mice have a striking reduction in the number of $\mathrm{CD}^{-} \mathrm{CD}^{-} \mathrm{CD} 25^{+}$ pro- $T$ cells in the thymus, where there is instead an expanded population of immature B cells.

How does lunatic fringe disrupt the balance of T- and B-lineage cells? To address this question, transgenic and wild-type bonemarrow cells were transferred together into irradiated Rag2 $/^{-}$mice, which lack lymphocytes and their precursors. Transgenic bone marrow gave rise to thymic B cells, as expected, but, surprisingly, so did the non-transgenic cells. This shows lunatic fringe can also act cell-non-autonomously. The authors also show that ectopic expression of lunatic fringe inhibits Notch1 activation in lymphoid progenitors

In these bone marrow chimaeras it is impossible to tell whether lunatic fringe promotes B-cell development merely by promoting their expansion, or by directly influencing the T- or B-cell-fate decision. To test this, lymphoid progenitors from norma mice were injected directly into the thymi of transgenic or non-transgenic mice, and their development was followed. In transgenic thymi, up to $90 \%$ of the non-transgenic lymphoid progenitors developed into Blineage cells and became mature B cells through the normal developmental programme.

The authors suggest that the choice of $T$ - versus B-cell fate can be influenced by the degree of Notch 1 activity in neighbouring progenitors, and ectopic lunatic fringe biases towards the B-cell fate in the thymus. Whether lunatic fringe has a physiological role in lineage commitment remains to be seen.

Jen Bell

(9) References and links ORIGINAL RESEARCH PAPER Koch, U. et al. Subversion of the $T / B$ lineage decision in the thymus by lunatic fringemediated inhibition of Notch-1. Immunity 15, 225-236 (2001) 
the thymi of Stat3 $3^{-/}$mice than in wild-type mice, and destruction of the thymic architecture was evident. When thymcytes were removed from the Stat 3 mutant mice and exposed in vitro to apoptotic stimuli, the cells were no more sensitive to apoptosis than thymocytes from wild-type mice. So, the increased sensitivity of these cells is not due to an intrinsic defect, but rather due to a failure of the Stat $^{-/-}$TECs to protect them from apoptotic stimuli.

In conclusion, these results show that Stat 3 is vital in the maintenance of thymic architecture and for the survival of thymocytes. As far as the thymus goes then, Stat 3 is a protein you need if you want to maintain control of the ageing process.

Jenny Buckland

(4) References and links ORIGINAL RESEARCH PAPER Sano, S et al. Stat 3 in thymic epithelial cells is essential for postnatal maintenance of thymic architecture and thymocyte survival. Immunity 15, 261-273 (2001) FURTHER READING

Anderson, G. \& Jenkinson, E. J. Lymphostromal interactions in thymic development and function. Nature Rev. Immunol. 1, 31-40

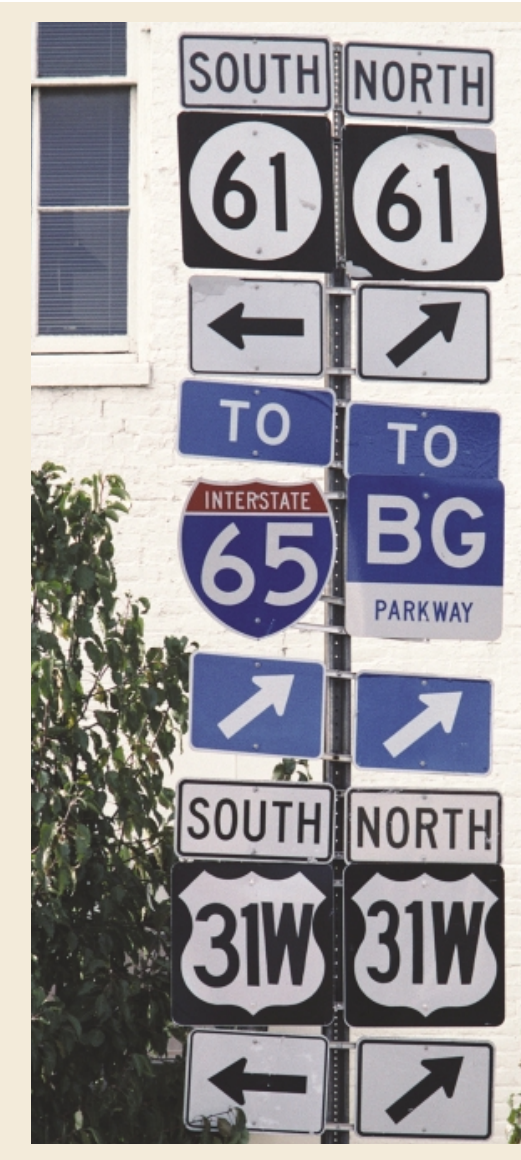

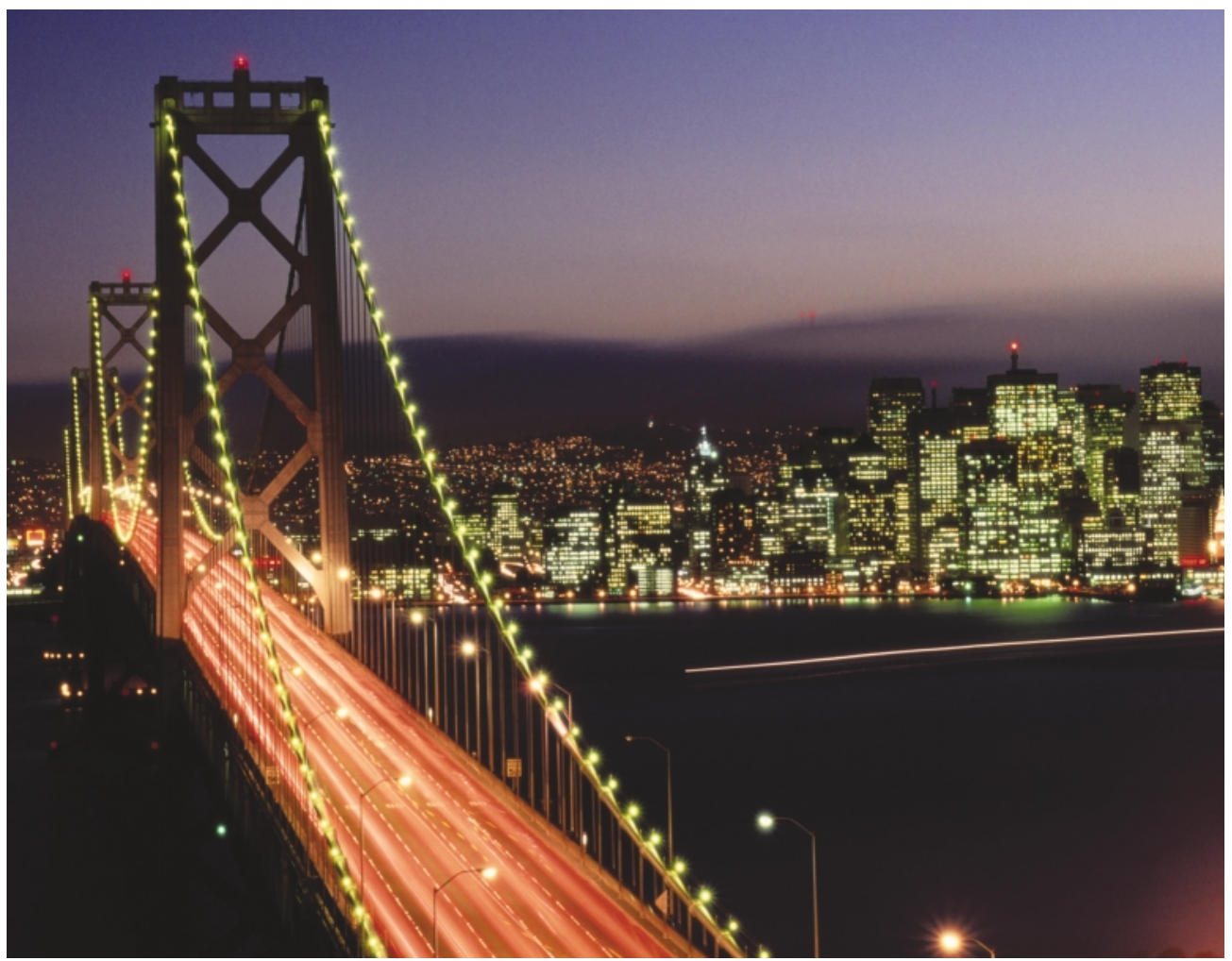

INNATE IMMUNITY

\section{New toll bridge}

The Toll-like receptors (TLRs) are emerging as the principal antigen sensors of innate immunity. In the earliest stages of infection, members of the TLR family discern the nature of the invader by detecting its conserved molecular patterns, and guide the ensuing adaptive response by inducing cytokines and costimulatory molecules. It is unclear how exactly this is achieved as all TLR-signalling components discovered to date are common to all TLRs and, indeed, interleukin-1 receptors (IL-1R). Two groups have now discovered a novel adapter molecule that specifically interacts with TLR4 and is a crucial bridge in the MyD88-independent signalling pathway.

MyD88 is a signalling adaptor containing a Toll/IL-1R (TIR) domain, a conserved motif that is essential for TLR signal transduction. Previous studies on MyD88-deficient mice, showed that this adapter is vital for signalling by TLRs, with the only known exception of TLR4 - a lipopolysaccharide (LPS) receptor - which can signal through both MyD88-dependent and MyD88-independent pathways. However, the adapter in this alternative, MyD88-independent pathway was not identified.

The new adaptor, described in the current studies, was identified in database searches for novel TIR domain-containing proteins. It is named TIR domain-containing adapter protein (TIRAP) by Horng et al., reporting in Nature Immunology; and MyD88-adapter-like (Mal), by Fitzgerald et al., reporting in Nature. Notably, unlike MyD88, TIRAP/Mal does not contain an amino-terminal death domain.

Both groups assessed TIRAP/Mal function in transfection studies, and showed that, similar to
MyD88, overexpression of TIRAP/Mal leads to activation of nuclear factor $\kappa \mathrm{B}(\mathrm{NF}-\kappa \mathrm{B})$. Is TIRAP/Mal, then, the elusive adapter of MyD88independent TLR signalling? To test this, both groups generated dominant-negative TIRAP/Mal mutants. The mutant TIRAP/Mal was shown to inhibit NF- $\kappa \mathrm{B}$ activation by TLR4, but did not interfere with NF- $\kappa$ B activation by TLR9 (the CpG receptor) or by IL-1R. Immunoprecipitation studies confirmed that TIRAP/Mal does indeed physically associate with TLR4.

To further investigate the functions of TIRAP/Mal, Horng and co-workers synthesized a peptide that corresponds with the predicted site of interaction with TLR4. This TIRAP/Mal peptide, attached to a delivery peptide that allows it to penetrate the cell membrane, inhibits LPS-induced, but not $\mathrm{CpG}$-induced, NF- $\kappa \mathrm{B}$ activation in a macrophage cell line. Furthermore, the TIRAP/Mal peptide was shown to inhibit LPS-induced, but not CpG-induced, upregulation of the co-stimulatory molecules CD80/CD86, cytokine production, and development of antigen-presenting capacity in dendritic cells.

Uncovering this new adapter is an important step in deciphering the complexity of TLR signalling, and determining which LPS-response genes are targets of MyD88- or TIRAP/Mal-dependent pathways must be a priority of future work.

(4) References and links

Jen Bell

ORIGINAL RESEARCH PAPERS Horng, T., Barton, G.M. \&

Medzhitov, R. TIRAP: an adapter molecule in the Toll signaling pathway. Nature Immunol. 2, 835-841 (2001) | Fitzgerald, K.A. et al. Mal (MyD88-adapter-like) is required for Toll-like receptor-4 signal transduction. Nature 413, 78-83 (2001)

FURTHER READING Akira, S., Takeda, K. \& Kaisho, T. Toll-like receptors: critical proteins linking innate and acquired immunity. Nature Immunol. 2, 675-680 (2001)

WEB SITES

Luke O'Neill's lab:

http://www.tcd.ie/Biochemistry/LONeill.html

Ruslan Medzhitov's lab:

http://info.med.yale.edu/immuno/fac_medzhitov.html 